\title{
No Consistent Relationship between Gamma Power and Peak Frequency in Macaque Primary Visual Cortex
}

\author{
Xiaoxuan Jia (贾哓轵), ${ }^{1 *}$ Dajun Xing (邢大军), ${ }^{3 *}$ and Adam Kohn ${ }^{1,2}$ \\ ${ }^{1}$ Dominick Purpura Department of Neuroscience and ${ }^{2}$ Department of Ophthalmology and Vision Sciences, Albert Einstein College of Medicine, Bronx, New \\ York 10461, and ${ }^{3}$ Center for Neural Science, New York University, New York, New York 10003
}

Neural activity in the gamma frequency range ("gamma") is elevated during active cognitive states. Gamma has been proposed to play an important role in cortical function, although this is debated. Understanding what function gamma might fulfill requires a better understanding of its properties and the mechanisms that generate it. Gamma is characterized by its spectral power and peak frequency, and variations in both parameters have been associated with changes in behavioral performance. Modeling studies suggest these properties are co-modulated, but this has not been established. To test the relationship between these properties, we measured local field potentials (LFPs) and neuronal spiking responses in primary visual cortex of anesthetized monkeys, for drifting sinusoidal gratings of different sizes, contrasts, orientations and masked with different levels of noise. We find that there is no fixed relationship between LFP gamma power and peak frequency, and neither is related to the strength of spiking activity. We propose a simple model that can account for the complex stimulus dependence we observe, and suggest that separate mechanisms determine gamma power and peak frequency.

\section{Introduction}

The gamma components $(30-80 \mathrm{~Hz})$ of the local field potential (LFP) reflect coordinated, rhythmic voltage fluctuations (Buzsaki, 2006), and have been found to be elevated in active brain states (Fries et al., 2001; Pesaran et al., 2002). Gamma has been proposed to play a role in stimulus feature binding (Gray et al., 1989; Singer, 1999; Engel et al., 2001) and to form a dynamic communication window for the interaction between neuronal populations (Engel et al., 2001; Fries, 2009), although these functions are debated (Roelfsema et al., 2004; Burns et al., 2010a,b, 2011; Chalk et al., 2010; Ray and Maunsell, 2010; Jia and Kohn, 2011).

The function gamma may fulfill depends in part on its power and peak frequency, and both of these properties correlate with behavioral performance (Womelsdorf et al., 2006; Edden et al., 2009). Understanding whether changes in gamma power and frequency are coupled is important for determining whether distributed groups of neurons with elevated gamma could be easily related to each other: to function as a binding or communication signal, the gamma frequencies in distributed neuronal groups need to be similar (and coherent) so that spikes associated with

Received April 5, 2012; revised Sept. 12, 2012; accepted Oct. 26, 2012

Author contributions:X.X.J., D.J.X., and A.K. designed research;X.X.J., D.J.X., and A.K. performed research; X.X.J., D.J.X., and A.K. analyzed data; X.X.J., D.J.X., and A.K. wrote the paper.

${ }^{*} X . X . J$. and D.J.X. contributed equally.

This work was supported by a grant from the National Institutes of Health to A.K. (EY016774) and Research to Prevent Blindness.

Correspondence should be addressed to either Adam Kohn or Xiaoxuan Jia, Department of Neuroscience, Albert Einstein College of Medicine, Kennedy Center Room 822, 1410 Pelham Parkway South, Bronx, NY 10461, E-mail: adam.kohn@einstein.yu.edu or jxiaoxuan@gmail.com; or to Dajun Xing, Center for Neural Science, New York University, 4 Washington Place, Room 809, New York, NY 10003, E-mail: xdj@cns.nyu.edu. D0I:10.1523/JNEUROSCI.1687-12.2013

Copyright $(2013$ the authors $\quad 0270-6474 / 13 / 330017-09 \$ 15.00 / 0$ those different rhythms will have a consistent temporal relationship (Atallah and Scanziani, 2009; Ray and Maunsell, 2010).

Models suggest gamma power is affected primarily by the strength of drive to inhibitory neurons or to a recurrently connected excitatory-inhibitory network (for review, see Bartos et al., 2007; Tiesinga and Sejnowski, 2009; and Whittington et al., 2011). The relationship between gamma power and peak frequency have been studied less extensively but previous work suggests that stronger drive, which produces more power, also yields higher frequencies (Whittington et al., 1995; Traub et al., 1996a, 1997; Buia and Tiesinga, 2006). Peak frequency can also be affected by synaptic kinetics and conductance (Whittington et al., 1995; Traub et al., 1996a, 1997; Wang and Buzsáki, 1996; Brunel and Wang, 2003), the balance between excitation and inhibition (Brunel and Wang, 2003), and cortical feedback (Kang et al., 2010).

The empirical relationship between gamma power and peak frequency in cortex is unclear. Ray and Maunsell (2010) found that raising stimulus contrast caused an increase in gamma power and peak frequency in primary visual cortex (V1), consistent with the suggestion that stronger drive leads to co-modulation of these properties. However, other studies have indicated little or no change in peak frequency with stimulus contrast (Henrie and Shapley, 2005, Bartolo et al., 2011), or have shown that stimulus manipulations that reduce power lead to a higher peak freqency (Gieselmann and Thiele, 2008; Lima et al., 2010; Bartolo et al., 2011). This has led to the suggestion that a critical determinant of frequency is the size of the activated network (but see Traub et al., 1996b; Gieselmann and Thiele, 2008; Schwarzkopf et al., 2012).

Here we provide a systematic evaluation of the relationship between gamma power and peak frequency in $\mathrm{V} 1$ of anesthetized macaque monkeys, by comparing responses for four different visual stimulus manipulations. We also compared changes in 
gamma to simultaneously recorded neuronal activity. We found a range of relationships between gamma power and its peak frequency, and a dissociation of both from neuronal response strength. We use a simple model to reproduce our physiological findings, and to probe potential underlying mechanisms.

\section{Materials and Methods}

Data were collected from 5 adult male monkeys (Macaca fascicularis). Animal preparation and general methods were similar to those described by Jia et al. (2011). Anesthesia was induced with ketamine $(10 \mathrm{mg} / \mathrm{kg})$ and maintained during surgery with isoflurane $\left(1.0-2.5 \%\right.$ in $\left.95 \% \mathrm{O}_{2}\right)$. Sufentanil citrate $(6-18 \mu \mathrm{g} / \mathrm{kg} / \mathrm{h}$, adjusted as needed for each animal) was injected intravenously to maintain anesthesia during recordings. Vecuronium bromide $(0.15 \mathrm{mg} / \mathrm{kg} / \mathrm{h})$ was used to suppress eye movements. All procedures were approved by the Albert Einstein College of Medicine at Yeshiva University and followed the guidelines in the United States Public Health Service Guide for the Care and Use of Laboratory Animals.

We implanted a $\sim 4 \times 4 \mathrm{~mm}$ array with 96 recording electrodes $(1 \mathrm{~mm}$ in length and $0.4 \mathrm{~mm}$ spacing) in the upper layers of V1. At each recording site, the raw voltage signal was filtered between $0.3 \mathrm{~Hz}$ and $250 \mathrm{~Hz}$ and sampled at $1 \mathrm{kHz}$ for LFP data, and between $250 \mathrm{~Hz}$ and $7.5 \mathrm{kHz}$ for spiking activity. Spiking activity that exceeded a user-defined threshold was digitized at $30 \mathrm{kHz}$ and sorted with Plexon Offline Sorter. Sorted units at each recording site were grouped together and treated as multiunit activity at that site. To minimize contamination by $60 \mathrm{~Hz}$ noise, we applied a fourth-order band-stop butterworth filter to the LFP data.

Visual stimuli were generated with custom software (EXPO) and presented on a calibrated CRT monitor $(100 \mathrm{~Hz}$ refresh rate) placed $110 \mathrm{~cm}$ from the monkey. Neuronal receptive fields were first mapped with small, drifting gratings $\left(0.6^{\circ} ; 250 \mathrm{~ms}\right.$ duration $)$ presented at a range of spatial positions. We then centered sinusoidal gratings of 1 cycle/degree drifting at 6.25 cycles/s on the aggregate receptive field. For experiments manipulating grating size ( $1-10^{\circ}$ diameter), stimuli were viewed monocularly. We observed similar effects when gratings were viewed binocularly, as for the remaining manipulations described below. Each size was presented at 16 different drift directions $\left(22.5^{\circ}\right.$ spacing; 30 repetitions per stimulus; $1 \mathrm{~s}$ presentations) at full contrast. For noise-masking experiments, large ( $10^{\circ}$ diameter $)$, full contrast gratings drifting in 16 different directions were masked with random spatial noise. Noise was generated by selecting small patches $\left(0.06 \times 0.06^{\circ}\right)$ from the original grating and computing their mean luminance. These patches were then placed at random locations on the image (Zhou et al., 2008). The proportion of the gratings replaced by these patches was defined as the noise level; noise was randomized on each frame. For manipulations of contrast, we used large gratings $\left(10^{\circ}\right)$ with contrast ranging from 0.01 to 1 , presented at 4 orientations $\left(45^{\circ}\right.$ steps). Each set of stimuli was presented in a pseudorandom sequence, with a gray screen interleaved to measure spontaneous activity. Not every stimulus manipulation was run in each animal.

Power spectra of the LFP were calculated using multi-taper methods with 8 tapers, for epochs 100-1000 ms after stimulus onset (Jia et al., 2011). Spiking responses were measured in identical epochs. Gamma power was calculated as the summed power in the $25-55 \mathrm{~Hz}$ range, after averaging spectra for individual stimulus orientations. Because LFP power decreases at higher frequencies, we defined peak frequency as the frequency of the maximum ratio between the spectra for stimulusinduced and spontaneous activity (Henrie and Shapley, 2005) between 25 and $55 \mathrm{~Hz}$. We obtained similar results when we used a frequency range of $20-100 \mathrm{~Hz}$, but the stimulus-dependent changes in gamma power were slightly smaller in magnitude and the changes in peak frequency slightly larger. Our analysis focused on power in the $25-55 \mathrm{~Hz}$ range because LFP components above $\sim 50 \mathrm{~Hz}$ behave similarly to local spike activity (Ray and Maunsell, 2011; Zanos et al., 2011; perhaps because of spectral contamination by the action potential waveforms) and differently from the low gamma activity that is our focus here. Calculating gamma power based on the ratio of the spectra for stimulus-driven and spontaneous activity also provided results similar to those we report.
Simulations. To gain insight into how different visual stimuli could modulate neuronal activity and the gamma components of the LFP, we constructed a simple model with three components (Fig. 1) - local excitatory $(E)$ and inhibitory $(I)$ components (Wilson and Cowan, 1972; Börgers and Kopell, 2008; Mazzoni et al., 2008) and a global (G) excitatory component (Kang et al., 2010). The local components can be thought of as a recurrently connected cortical hypercolumn or set of such columns; the $G$ component can be thought of as a more global feedback, which might involve long-range horizontal connections or feedback from higher cortex (see also Results).

The dynamic interactions among $E, I$, and $G$ are described by Equations $1-3$. The interaction strengths are denoted by $W_{\mathrm{SR}}$, where $S$ denotes the sender and $R$ the receiver; the interaction type (excitatory or inhibitory) is denoted by the sign of $W_{\mathrm{SR}}$ (positive or negative):

$$
\begin{array}{r}
\tau_{E} \frac{d E}{d t}=-E+W_{E E} \cdot[H(E) \cdot(1-\mathrm{MN})]-W_{I E} \cdot H(I) \\
+W_{G E} \cdot H(G)+I_{E}(c, \mathrm{MN}, \theta) \\
\tau_{I} \frac{d I}{d t}=-I+W_{E I} \cdot[H(E) \cdot(1-\mathrm{MN})]-W_{I I} \cdot H(I) \\
+W_{G I} \cdot H(G)+I_{I}(c, \mathrm{MN}, \theta) \\
\tau_{G} \frac{d G}{d t}==-G+W_{E G} \cdot\left[H(E) \cdot(1-\mathrm{MN}) \cdot r^{2}\right]
\end{array}
$$

where

$$
H(x)=\begin{array}{ll}
x, & \text { if } x>0 \\
0, & \text { otherwise }
\end{array}
$$

and

$$
I_{E}(c, \mathrm{MN}, \theta)=R_{\max } \cdot \frac{\mathrm{c}^{2}}{\mathrm{c}^{2}+\mathrm{c}_{50}^{2}} \cdot(1-\mathrm{MN}) \cdot\left(1+(\cos \theta)^{2}\right) / 2 .
$$

with $I_{\mathrm{I}}$ defined similarly. The parameter MN represents the level of masking noise; $r, c$, and $\theta$ represent the stimulus radius, contrast and orientation, respectively. Other parameters are explained below.

Thus, in our model the local $E$ component connects to the local $I$ component with coupling strength $W_{\mathrm{EI}}$, and to itself with strength $W_{\mathrm{EE}}$; similarly, the local $I$ component connects to $E$ with strength $W_{\text {IE }}$ and to itself with $W_{\text {II }}$. The $E$ component connects to the $G$ component with strength $W_{\mathrm{EG}}$, and this is weighted by the size of the stimulus (i.e., by the square of its radius, $r$ ), a simple instantiation of pooling over multiple, local $E$ components. $G$ provides input to $E$ and $I$ with coupling strength $W_{\mathrm{GE}}$ and $W_{\mathrm{GI}}$, respectively.

The $\tau_{\mathrm{E}}, \tau_{\mathrm{I}}$, and $\tau_{\mathrm{G}}$ in Equations $1-3$ are the time constants for $E, I$, and $G$, respectively. Equation 4 implements a simple instantiation of a "spike" threshold: only values of $E, I$, and $G$ that exceed threshold (i.e., that are greater than zero) will affect other neurons.

Both $E$ and $I$ receive independent inputs $\left(I_{\mathrm{E}}\right.$ and $I_{\mathrm{I}}$, respectively) that are functions of stimulus contrast $(c)$, orientation $(\theta)$, and masking noise (MN) (Eq. 5). The value of $I_{\mathrm{E}}$ and $I_{\mathrm{I}}$ define the mean of a Poisson distribution, from which we drew a random variable on each time step. The orientation tuning of the input included a prominent untuned component, so that all orientations provided substantial input. The contrast dependence on the input was implemented with a hyperbolic ratio function (Albrecht and Hamilton, 1982), where $R_{\max }$ is an arbitrary scaling factor that sets the peak strength of the input and $c_{50}$ defines the contrast sensitivity.

We solved the equations numerically, with a time resolution of $0.1 \mathrm{~ms}$ using the Euler method. We ran the model for $1.6 \mathrm{~s}$ with 50 repeats for each stimulus condition. We analyzed responses starting $500 \mathrm{~ms}$ after response onset, to ensure the network had settled to a steady state. We used the average half-rectified value of $E$ (i.e., $(H(E))$ as a measure of the 


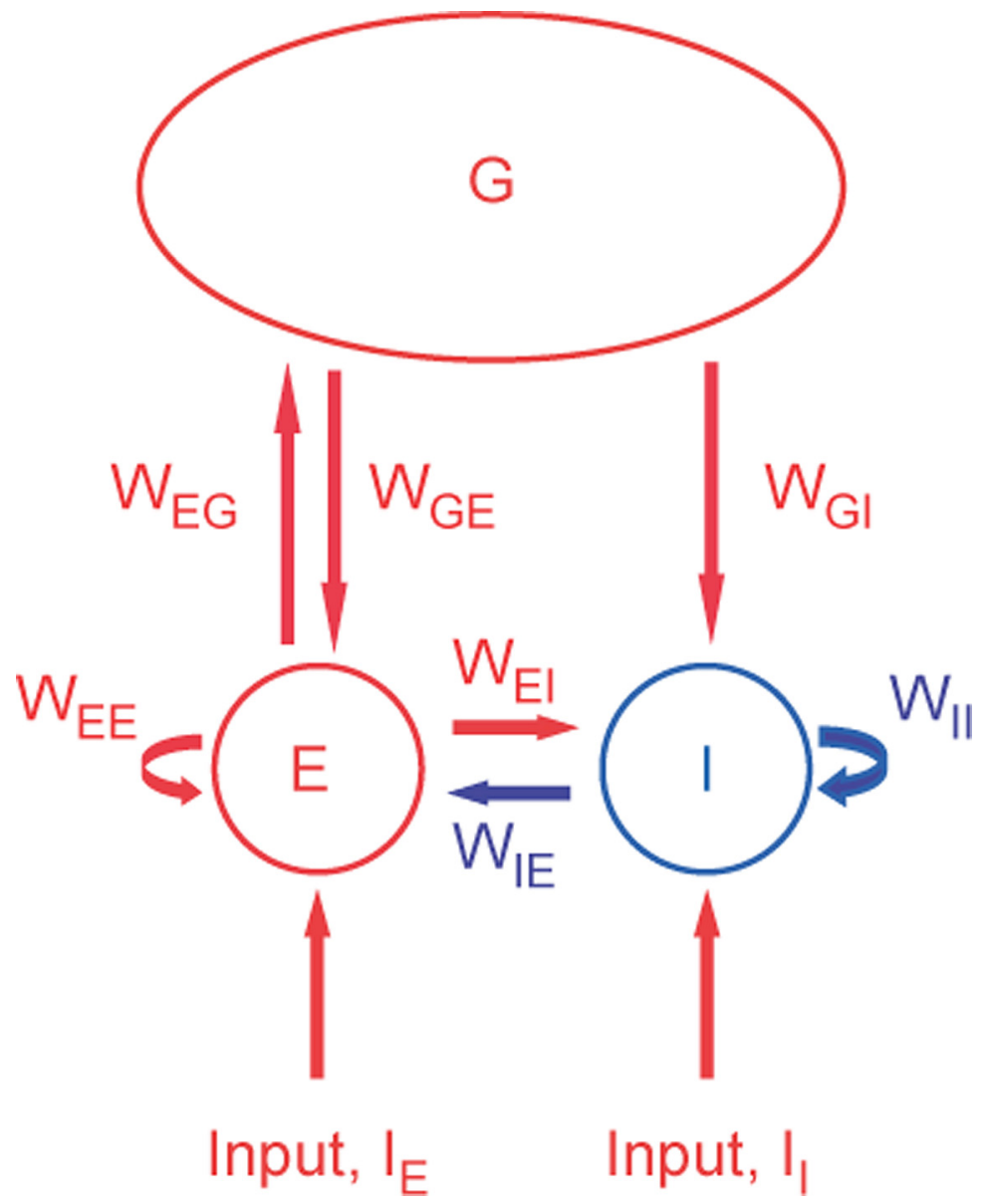

Figure 1. Architecture of the model. The model consisted of local excitatory $(E)$ and inhibitory $(I)$ components and a global $(G)$ component. The local components provided input to each other $\left(W_{\mathrm{El}}\right.$ and $\left.W_{\mathrm{IE}}\right)$ and to themselves $\left(W_{\mathrm{EE}}\right.$ and $\left.W_{\mathrm{II}}\right)$. The global component was driven by the $E$ component $\left(W_{\mathrm{EG}}\right)$, and provided excitation to both $E$ and $/\left(W_{\mathrm{GE}}\right.$ and $W_{\mathrm{GI}}$, respectively). Both $E$ and I were driven by external input, $I_{\mathrm{E}}$ and $I_{1}$. Red indicates excitatory input or connections; blue indicates inhibition.

\section{Results}

We measured neuronal firing rates and LFP gamma power and peak frequency in V1 of 5 anesthetized macaque monkeys, for drifting sinusoidal gratings of different sizes, contrasts, orientations and masked with different levels of noise.

We first determined how gamma was affected by grating size, ranging from 1 to $10^{\circ}$ in diameter. We analyzed a common set of sites across conditions-those that were activated by the smallest grating. The average LFP spectra showed nearly a twofold increase in gamma power with larger gratings (Fig. $2 \mathrm{~A}$, left), with a more apparent bump in the gamma range. We quantified the change in gamma power (25-55 $\mathrm{Hz}$ ) by normalizing to its maximum across stimulus sizes at each site, and then averaging across sites ( $n=209$ sites). This normalized gamma power increased monotonically from $0.67 \pm 0.02$ for the smallest grating to $0.93 \pm 0.01$ for the largest $(p<0.0001$, Wilcoxon signedrank test; Fig. $2 \mathrm{~A}$, middle, black). Similar effects were seen in each individual animal (faint black lines, indicating average effect in each animal). Over the same range of sizes, the gamma peak frequency at individual recording sites decreased from $50.5 \pm$ 0.2 to $37.9 \pm 0.1 \mathrm{~Hz}(p<0.0001, t$ test; Fig. $2 A$, middle, red; data from each animal in faint red). Thus, gamma power and peak frequency were modulated in opposite ways by stimulus size: an increase in gamma power was associated with a decrease in peak frequency. The simultaneously re-

Table 1. Parameters in the three-component model

\begin{tabular}{llll}
\hline & $E$ & $I$ & $G$ \\
\hline$\tau$ (seconds) & 0.006 & 0.015 & 0.019 \\
Coupling from $E$ to & $W_{\mathrm{EE}}=1.5$ & $W_{\mathrm{EI}}=3.5$ & $W_{\mathrm{EG}}=0.6$ \\
Coupling from $/$ to & $W_{\mathrm{IE}}=3.25$ & $W_{\mathrm{II}}=2.5$ & $\mathrm{~N} / \mathrm{A}$ \\
Coupling from $\mathrm{G}$ to & $W_{\mathrm{GE}}=0.25$ & $W_{\mathrm{GI}}=0.5$ & $\mathrm{~N} / \mathrm{A}$ \\
\hline
\end{tabular}

N/A, Not applicable.

mean firing rate, and the power and peak frequency in the spectrum of $E$ as measures of the power and peak frequency of the LFP.

Parameters. The values of coupling strengths and $\tau \mathrm{s}$ are provided in Table 1. We assumed that local $E$ has the fastest time constant $(6 \mathrm{~ms})$ and the time constant for local $I$ and global $G$ are slower ( 15 and 19 $\mathrm{ms}) . R_{\max }$ and $c_{50}$ were set to 40 and 0.3 , respectively, for $I_{\mathrm{E}}$, and to 32 and 0.3 for $I_{\mathrm{I}}$. These parameters were fixed for all simulations of stimulus manipulations.

Variations in stimulus conditions were implemented by altering the values of $r, c, \theta$, and $\mathrm{MN}$. In simulations of the size experiment, we set $\mathrm{MN}=0, c=1, \theta=0$ and varied $r$ from 1 to 5 . For the contrast experiments, we used $\mathrm{MN}=0, r=5, \theta=0$ and varied $c$ from 0.0156 to 1 . For the orientation experiments, we used $\mathrm{MN}=0, r=5, c=1$ and varied $\theta$ from 0 to $90^{\circ}$. For the noise experiment, we used $r=5, c=1, \theta=0$ and varied $\mathrm{MN}$ from 0 to 0.6 . The range of $\mathrm{MN}$ was chosen to match the physiological data; the magnitude of $\mathrm{MN}$ has no direct relationship to the proportion of the grating replaced by noise. corded neuronal responses showed strong suppression for large gratings, with the normalized spike rate decreasing from $0.86 \pm 0.02$ to $0.41 \pm 0.02(p<0.0001$, Wilcoxon signed-rank test; Fig. $2 A$, right).

We next measured gamma induced by large gratings $\left(10^{\circ}\right)$ masked with different levels of noise. Noise was generated by replacing different proportions of the gratings with random large pixels of the same mean luminance (see Materials and Methods). We used large gratings because these induced the most gamma power, for which the peak frequency was most clearly defined. Masking noise reduced gamma power (see also Jia et al., 2011), and caused the peak frequency of the average spectrum to shift lower (Fig. 2 B, left; $n=228$ sites). Across individual sites, normalized gamma power fell twofold when gratings were masked with $80 \%$ noise, and the average peak frequency shifted from $42.2 \pm 0.1 \mathrm{~Hz}$ to $30.1 \pm 0.4 \mathrm{~Hz}(p<0.0001, t$ test; Fig. $2 B$, middle). Thus, for masking noise, gamma power and peak frequency were positively correlated across stimulus conditions: a decrease in gamma power was associated with a decrease in peak frequency. Despite prominent changes in gamma, population neuronal firing rates were not affected by noise masking (ANOVA: $F=1.18, p=0.14$; Fig. $2 B$, right). The normalized spike rate for the unperturbed gratings was indistinguishable from the $80 \%$ noise condition $(0.79 \pm 0.02$ vs $0.78 \pm 0.01 ; p=$ 0.04, Wilcoxon signed-rank test). 
A
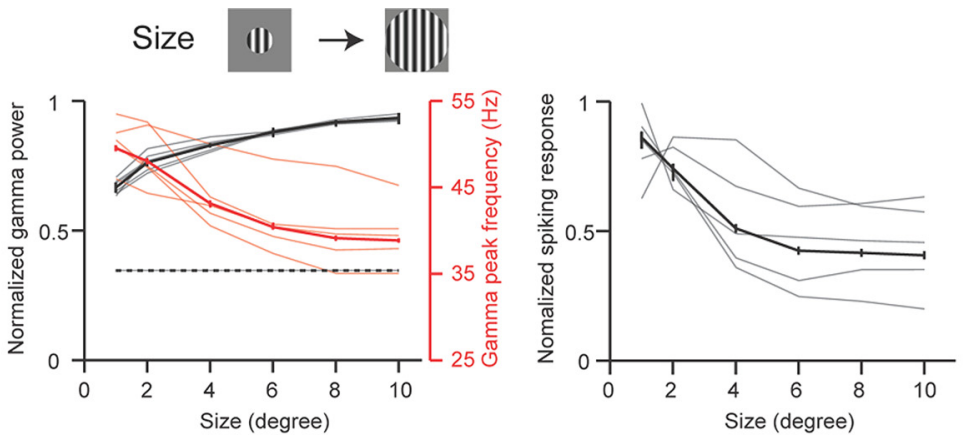

B

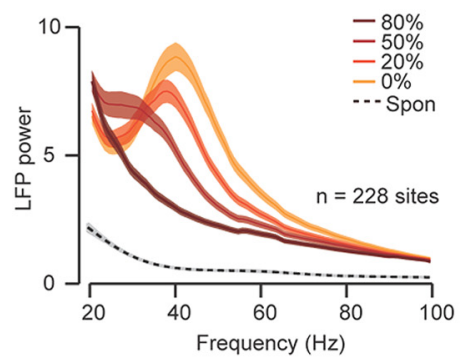

C

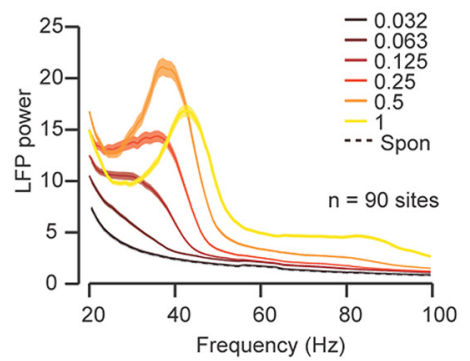

D

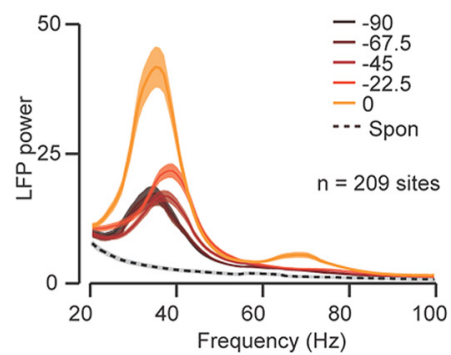

Noise-masking
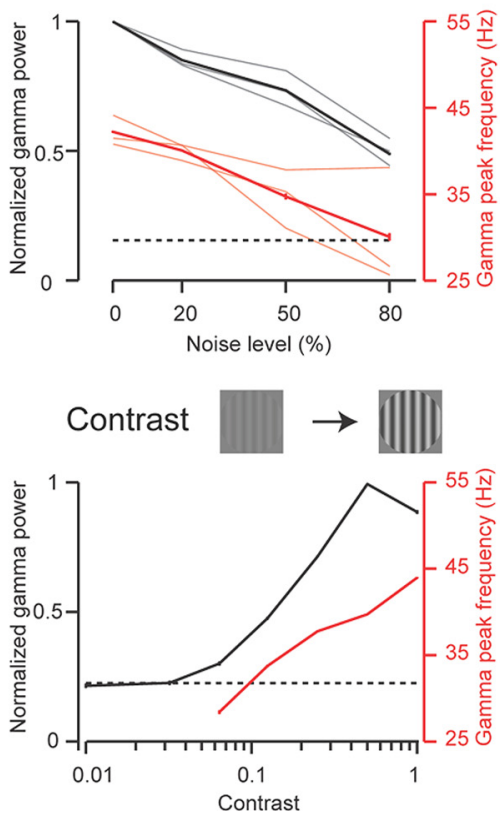

Orientation

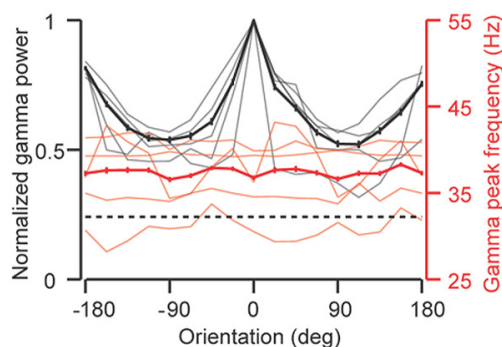

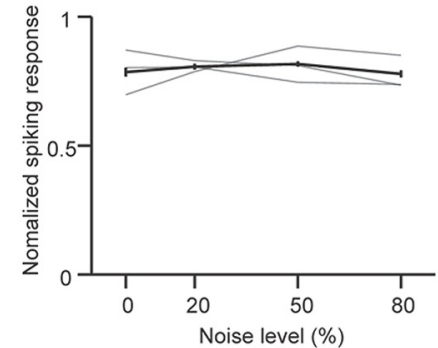
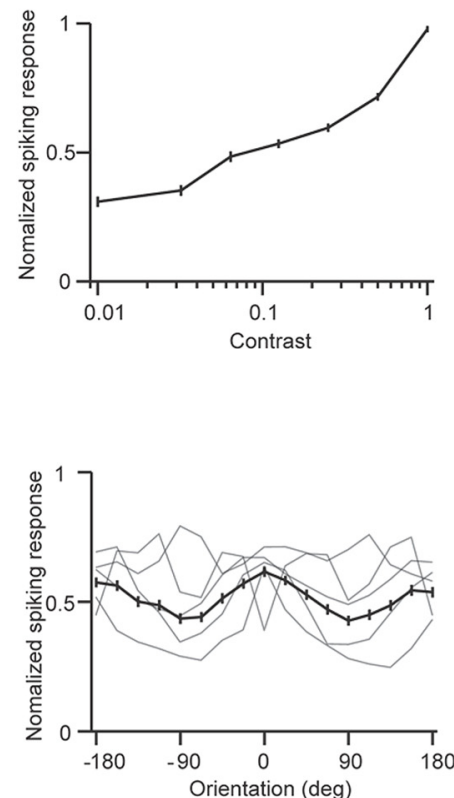

Figure 2. Gamma power, peak frequency, and neuronal firing rate for different stimulus manipulations in V1. A, Left, Power spectra of LFP for gratings of different sizes ( $n=209$ sites). Dashed line indicates the gamma power for spontaneous activity. Middle, Peak frequency in the gamma range (thick red line) and normalized gamma power (thick black line). The faint lines indicate the average data from each animal. Dashed line indicates the gamma power for spontaneous activity. Right, Normalized neuronal responses (thick black line). Faint lines indicate the average data from each animal. $\boldsymbol{B}$, Left, Power spectra of LFP for different levels of noise-masking ( $n=228$ sites). Middle, Peak frequency and normalized gamma power. Right, Normalized neuronal firing rate. C, Left, Power spectra of LFP for different stimulus contrasts ( $n=90$ sites). Middle, Peak frequency and normalized gamma power. Right, Normalized neuronal responses. $\boldsymbol{D}$, Left, Power spectra of LFP for gratings of different orientations ( $n=209$ sites). Middle, Peak frequency and normalized gamma power. Right, Normalized neuronal responses. All error bars indicate SEM.

As with masking noise, gamma power and peak frequency were positively correlated for manipulations of stimulus contrast. Increasing the contrast of a large grating $\left(10^{\circ}\right.$ in diameter $)$ enhanced both gamma power and peak frequency (Fig. 2C, left; $n=$ 90 sites). Since there was no induced gamma power when stimulus contrast was 0.01 or 0.032 , no peak frequency was estimated for those conditions. As stimulus contrast increased from 0.064 to 1 , the peak frequency increased from $28.4 \pm 0.2$ to $44.0 \pm 0.1$
$\mathrm{Hz}(p<0.0001, t$ test $)$, and stimulus-induced gamma power was enhanced approximately fourfold ( $p<0.0001$; Fig. $2 C$, middle). As stimulus contrast increased from 0.01 to 1 , the normalized spike rate increased $>3$-fold, from $0.31 \pm 0.02$ to $0.98 \pm 0.01$ $(p<0.0001$, Wilcoxon signed-rank test; Fig. $2 C$, right).

Finally, we measured the orientation tuning of gamma power and peak frequency and compared these to neuronal spiking activity. We used responses to large gratings $\left(10^{\circ}\right)$ drifting in 16 

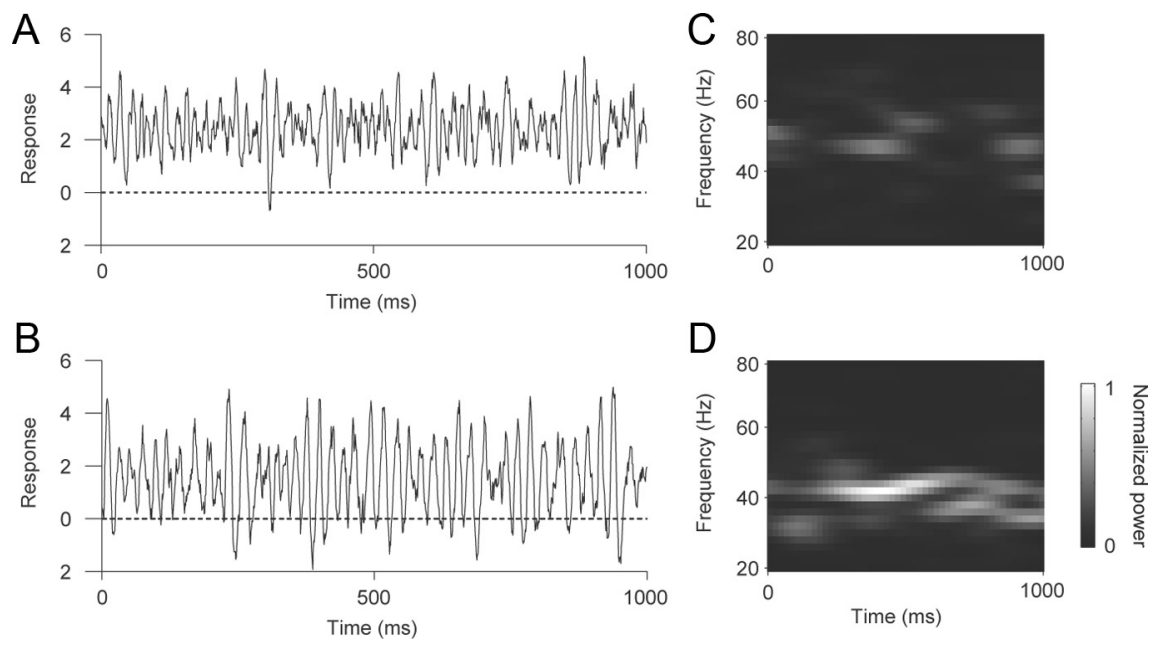

Figure 3. Example simulation responses. $A$, Response of the $E$ component to a small grating $(r=3)$, as a function of time. Note the strong mean response, and the presence of transient gamma fluctuations. Smaller gratings ( $r=1$ or 2$)$ produced stronger responses, but too little gamma power to visualize in a single trial. $\boldsymbol{B}$, Response of the $E$ component to a large grating $(r=5)$. Note the decrease in mean response and enhancement of gamma band activity. $\boldsymbol{C}, \boldsymbol{D}$, Spectrogram of the epochs shown in $\boldsymbol{A}$ and $\boldsymbol{B}$, respectively. Gamma activity is weaker and at a higher frequency for the small grating. Spectra were computed in a sliding $512 \mathrm{~ms}$ window, centered at the time indicated; spectra were smoothed for display only, by convolving a two-dimensional Gaussian kernel with the data.

different directions (conditions averaged in Fig. 2 A). To quantify across recording sites, we defined the preferred orientation (that inducing maximal gamma power) at each site to be $0^{\circ}$. The average power spectra for a subset of orientations are shown in Figure $2 D$ (left; same $n=209$ sites as in Fig. 2A). On average, gamma power at the preferred orientation was approximately twice that of the orthogonal orientations. However, the peak frequency of gamma was not significantly modulated by stimulus orientation, with the peak frequency at the preferred and orthogonal orientations nearly indistinguishable $(36.8 \pm 0.3$ vs $36.6 \pm 0.3 \mathrm{~Hz}, p=$ $0.6, t$ test; Fig. $2 D$, middle). When arranged by the preferred orientation of gamma power, spike rate tuning functions showed a response at the preferred orientation that was significantly $<1$, indicating that the preferred orientation defined by spike rates was often different from gamma at individual sites (Berens et al., 2008; Jia et al., 2011).

\section{Model of gamma generation}

We simulated the stimulus dependence of spiking activity and gamma power and peak frequency with a simple three component model, an extended but simplified version of the model developed by Kang et al. (2010). The model consisted of a local excitatory $(E)$, local inhibitory $(I)$, and global $(G)$ excitatory component (see Fig. 1 and Materials and Methods). The local $E$ and $I$ components represent populations in a local V1 region, such as in a cortical hypercolumn. The local $E$ and $I$ components were recurrently connected-the excitatory component provided input to the inhibitory component $\left(W_{\mathrm{EI}}\right)$ and to itself $\left(W_{\mathrm{EE}}\right)$, and similarly for the inhibitory component $\left(W_{\mathrm{IE}}\right.$ and $W_{\text {II }}$ ). Both the $E$ and $I$ components also received external and independent Poisson-distributed input $\left(I_{\mathrm{E}}\right.$ and $\left.I_{\mathrm{I}}\right)$. This architecture captures the basic pyramidal-interneuron network gamma (PING) model, commonly used to model gamma generation (Bartos et al., 2007; Tiesinga and Sejnowski, 2009; Whittington et al., 2011).

The third component-the global or $G$ component-represents a more spatially extensive mechanism. The global component integrates excitatory activity in multiple local regions
$\left(W_{\mathrm{EG}}\right)$, and affects both the local $E$ and $I$ components through excitatory connections ( $W_{\mathrm{GE}}$ and $W_{\mathrm{GI}}$, respectively). $G$ might arise from long-distance horizontal connections between columns in $\mathrm{V} 1$ or feedback from higher visual areas (Angelucci and Bresslof, 2006). Figure 3, $A$ and $B$, shows the behavior of the $E$ component of the model for a small and large high contrast "gratings" (i.e., input), respectively. The mean activity of $E$ is higher for the smaller stimulus. Spectral analysis shows the presence of elevated gamma frequency components in the activity of $E$, which are weaker and at a higher peak frequency for small gratings (centered around $50 \mathrm{~Hz}$, Fig. 3C) than large gratings $(\sim 40 \mathrm{~Hz}$, Fig. $3 \mathrm{D})$. The peak frequency of gamma shifts lower as power increases because the time constant for the global component, recruited more strongly by the large stimulus, is slower than for the local $E$ and $I$, lowering the resonant frequency of the network. Note that for both simulations gamma power fluctuates in time, consistent with previous analysis of physiological data (Burns et al., 2010a,b, 2011).

To compare our simulations with our physiological data, we equated the half-rectified mean activity of $E$-which we refer to as response strength - with measured firing rate. We did so because most neurons in cortex are excitatory and extracellular recording techniques are biased toward spikes arising from larger neurons (i.e., excitatory pyramidal cells); the behavior of I was qualitatively similar, except as noted below. We quantified the total power in the gamma range $(25-55 \mathrm{~Hz})$ and the gamma peak frequency for the $E$ component, as a measure of the LFP. Figure $4 A$ shows the behavior of these three variables for a range of stimulus sizes. The model produces a decrease in response strength (approximately a twofold decrease) and gamma peak frequency (approximately a $10 \mathrm{~Hz}$ decrease), and an increase in gamma power (a near doubling), as observed in our physiological data.

We simulated our dynamic noise-masking results by altering input strength and excitatory coupling. This captured the strong decrease in gamma power and peak frequency with higher levels of noise masking, with a nearly maintained response strength (Fig. 4B). This stimulus manipulation was the most difficult to simulate, and required reducing external input and excitatory coupling (to both the $E$ and $I$ components), but not inhibitory coupling. This might reflect that excitatory synapses are more prone to synaptic depression than many inhibitory ones during high-frequency or transient drive (Thomson and Deuchars, 1997; Gupta et al., 2000), such as that likely provided by the dynamic noise we used. Note that although we reduced the strength of excitatory input and recurrent excitation, response strength decreased only slightly ( $18 \%$ decrease in Fig. $4 B)$. This is because these manipulations reduced the inhibitory response strongly ( $\sim 40 \%$ over the same range), largely offsetting the loss of excitation. Implementing the same manipulation in a model with weaker recurrent inhibition (smaller values of $W_{\text {IE }}$ and $W_{\text {II }}$ ) produced a strong loss of response strength (data not shown).

To simulate the effect of manipulating the contrast of a large grating, we altered the strength of external input to the network 
A Size ili $\rightarrow$ ||||||
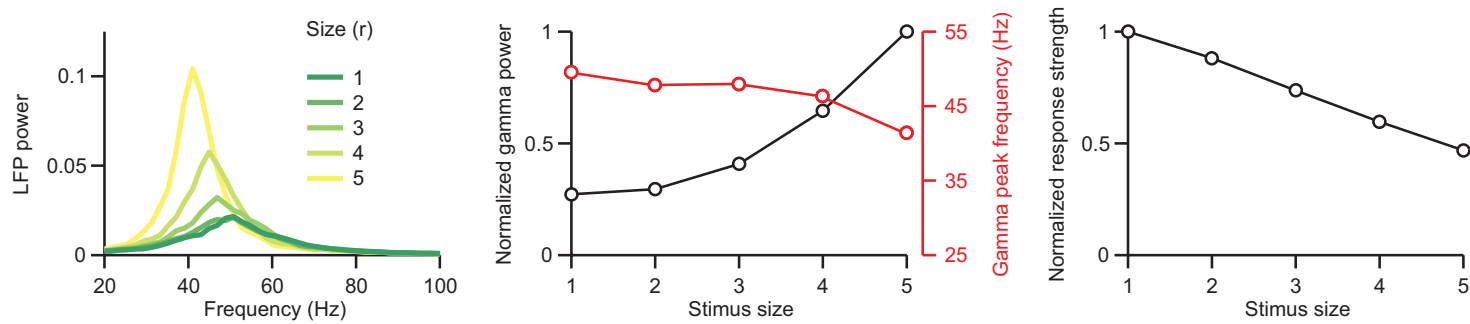

B

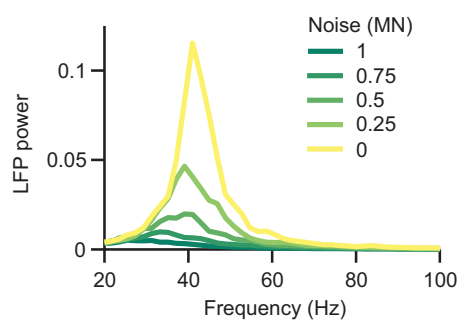

C

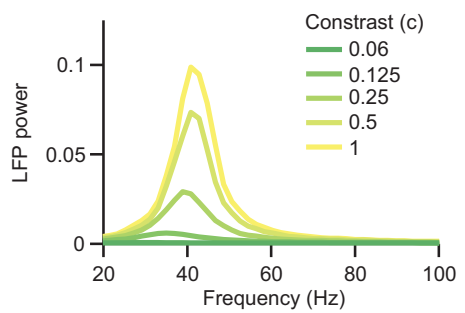

D

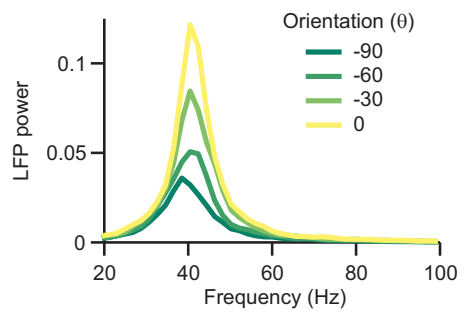

Noise-masking

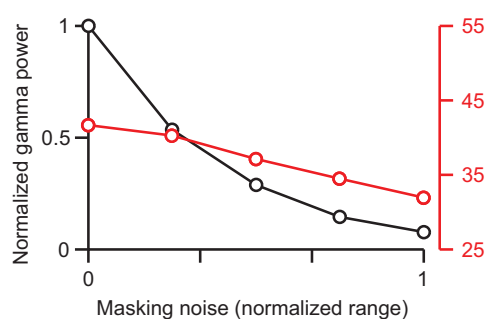

Contrast
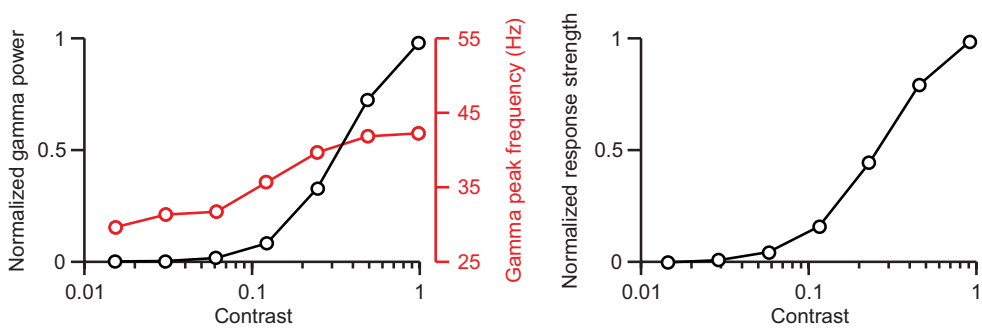

\section{Orientation}

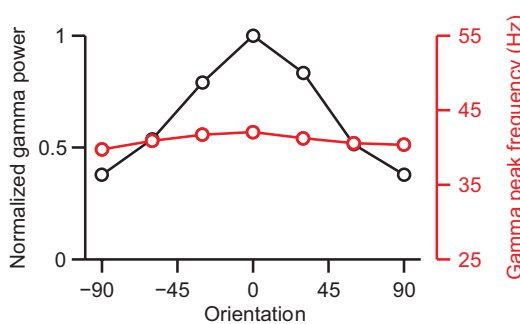

Figure 4. Simulated gamma power, peak frequency and neuronal fring rate for different stimulus manipulations. $A$, Left, Power spectra of LFP for gratings of different sizes Middle, Peak frequency in the gamma range (red) and normalized gamma power (black). Right, Normalized neuronal responses. B, Left, Power spectra of LFP fordifferentlevels ofnoise-masking. Middle, Peak frequencyand normalized gamma power. Right, Normalized neuronal firing rate. C, Left, Power spectra of LFP for different stimulus contrasts. Middle, Peak frequency and normalized gamma power. Right, Normalized neuronal responses. D, Left, Power spectra of LFP for gratings of different orientations. Middle, Peak frequency and normalized gamma power. Right, Normalized neuronal responses.

( $I_{\mathrm{E}}$ and $I_{\mathrm{I}}$; see Materials and Methods). At higher contrasts, input was stronger. Under this regime, the model produced higher firing rates and stronger gamma power with a higher peak frequency (Fig. 4C), as observed physiologically (Fig. 2C). A similar behavior has been observed in PING networks, in which power and peak frequency both increase with input drive (Whittington et al., 2011). This occurs because gamma fluctuations reflect the reciprocal interaction between the excitatory and inhibitory populations: excitation recruits inhibition which dampens excitation. As the drive to the network increases, this cyclic dynamic is enhanced and sped up. In our model this is also true, but this mechanism alone produces relatively small changes in peak frequency $(\sim 2 \mathrm{~Hz})$. The shift in peak frequency in our model is enhanced by the $G$ component. As input weakens, the influence of the $G$ component is stronger relative to the local $E$ and $I$ components, shifting the resonant frequency lower. Our model would thus predict that manipulating the contrast of a small grating-for which the influence of the $G$ component is substantially lower-would have a weak effect on peak frequency (see also below). This may explain different reported effects of altering stimulus contrast on gamma peak frequency (see Discussion). 


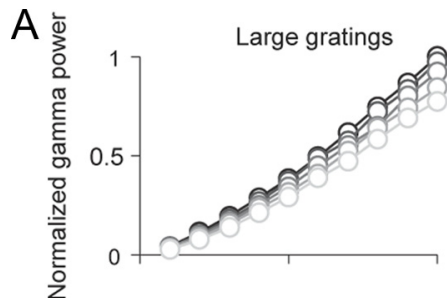

D

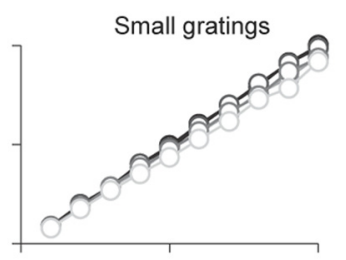

$\tau$ scaling factor $-1$ -1.1
-1.2 -1.3
-1.4 B

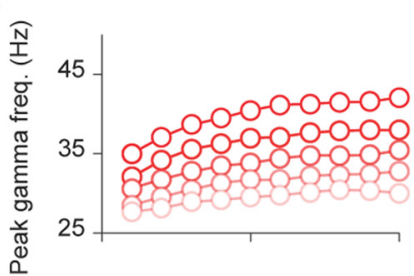

E

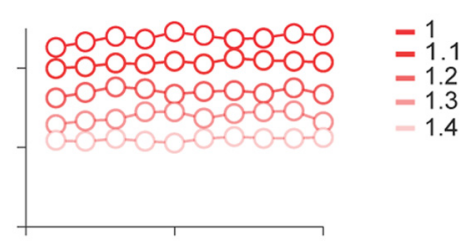

C

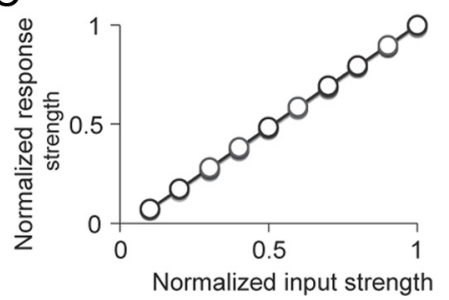

$\mathrm{F}$

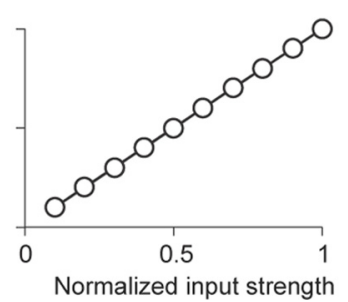

$\tau$ scaling

factor

-1
-11

$-1.2$

$-1.3$

$-1.4$

Figure 5. Dependence of model gamma power and peak frequency on input strength and time constants. $\boldsymbol{A}$, Gamma power as a function of input strength for a large grating $(r=5)$. Input strength of 1 corresponds to values of $R_{\max }=40, c=1, \mathrm{MN}=0, \theta=0$ for $I_{\mathrm{E}}$, as in Equation $5 ; R_{\max }=32$ for $I_{1}$. Weaker inputs were provided by scaling $I_{\mathrm{E}}$ and $I_{1}$ by the values indicated on the abscissa. Different line shades correspond to simulations in which the model time constants $(\tau)$ were multiplied by the factor indicated. $\boldsymbol{B}$, Same as $\boldsymbol{A}$ but for gamma peak frequency. $\boldsymbol{C}$, Same as $\boldsymbol{A}$ but for response strength. $\boldsymbol{D}-\boldsymbol{F}$, Same as $\boldsymbol{A}-\boldsymbol{C}$ but for small gratings $(r=1)$.

We simulated the effect of altering the orientation of a large grating by assuming an orientation bias in the input to local $E$ and $I$ (Eq. 5), consistent with our previous work suggesting the orientation tuning of gamma may reflect a weak bias for some stimulus orientations in a region of V1 (Jia et al., 2011). We defined the input to be maximal for an orientation of $0^{\circ}$. Figure $4 D$ shows that as the stimulus orientation deviates from $0^{\circ}$, there is a decrease in gamma power and firing rate but gamma peak frequency is unaltered, consistent with our physiological results.

Note that manipulations of stimulus contrast and orientation both involve only altering the strength of input to the network. However, lowering contrast caused both gamma peak frequency and power to decrease, whereas manipulations of stimulus orientation altered power but left peak frequency unaffected. This is because power is more sensitive to a reduction in input than peak frequency. Power decays linearly with weaker input to model (both $I_{\mathrm{E}}$ and $I_{\mathrm{I}}$; black line in Fig. $5 \mathrm{~A}$ ); peak frequency shows evidence of saturation with input strength and only changes substantially when input is reduced more than twofold (red line in Fig. $5 B$ ). Unlike the simulations, our physiological data show that power and peak frequency are similarly sensitive to reducing stimulus contrast (Fig. 2C; see also Ray and Maunsell, 2010). Thus the simulations capture the difference in gamma power and peak frequency for low and full contrast stimuli, but not how these properties are altered by smaller manipulations of contrast.

We explored an alternative manipulation to account for the sensitivity of gamma to stimulus contrast. Decreasing stimulus contrast is known to slow neuronal response dynamics and latency in V1 (Gawne et al., 1996; Carandini et al., 1997), an effect that can be captured by an increase in the neuronal membrane time constants (Carandini et al., 1997). We therefore determined the effect of lengthening the time constants in our model $\left(\tau_{\mathrm{E}}, \tau_{\mathrm{I}}, \tau_{\mathrm{G}}\right)$ by a scaling factor that varied from 1 to 1.4. This had the minimal effect on gamma power (increasingly faint lines in Fig. $5 \mathrm{~A}$ ) but caused a dramatic reduction in gamma peak frequency (increasingly faint lines in Fig. 5B). Response strength (mean value of $E$ component) was not affected by this manipulation (Fig. 5C). Thus, if reducing stimulus contrast causes both a weaker input drive and a slight slowing of dynamics, both power and peak frequency would be expected to decrease as contrast is reduced.

For comparison, the corresponding results for lowering the contrast of a small grating are shown in Figure 5D-F. As mentioned previously, small gratings do not generate strong activity in the $G$ component and, as a result reducing input, has a minimal effect on peak frequency (horizontal lines in Fig. 5E). Altering network time constants, on the other hand, does reduce the peak frequency (increasingly faint red line in Fig. $5 E$ ) and has minimal effect on power (Fig. 5D). Thus, for large gratings weaker input strength and slower network time constants both reduce power, whereas for small gratings only the latter contributes.

\section{Discussion}

We tested the relationship between LFP gamma power and peak frequency for four different visual stimulus manipulations. Increasing grating size induced a stronger gamma rhythm with a lower peak frequency, and suppressed neuronal responses. Lowering contrast or adding masking noise reduced gamma power and lowered the peak frequency; spike rates fell significantly as contrast was lowered, but showed no noticeable change with noise masking. Stimulus orientation strongly modulated gamma power but had little effect on peak frequency. Thus, our results show that gamma power does not have a fixed relationship to its peak frequency, nor to neuronal firing rates. An increase in gamma power may be associated with an increase (contrast and masking), decrease (size) or no change (orientation) in peak frequency, and with either an increase (contrast), decrease (size) or no change (masking and orientation) in neuronal response strength.

Our observations of how gamma is affected by stimulus size are consistent with those of Gieselmann and Thiele (2008) and Ray and Maunsell (2011). Our measurements with stimulus contrast are consistent with those of Ray and Maunsell (2010), but not with two other studies which showed that higher contrast stimuli induced more gamma power without any indication of a shift in peak frequency (Henrie and Shapley, 2005; Bartolo et al., 2011). This may be due to their use of smaller stimuli, which induce a broadband increase in power but a weaker gamma spectral "bump" (Jia et al., 2011). Our model shows that shifts in peak frequency should be smaller when the network is driven with a small stimulus, so long as the weakened input is not accompanied by a prominent reduction in network time constants. Consistent with the suggestion that gamma is strongly influenced by a global, integrative mechanism, gamma is particularly strong in the superficial layers (Xing et al., 2012) where the influence of long-range horizontal connections and feedback is prominent (Angelucci and Bresslof, 2006). 
Previous studies have shown that gamma power is orientation selective (Frien et al., 2000; Berens et al., 2008; Jia et al., 2011), without reporting whether differences in power are accompanied by systematic changes in peak frequency. Our results show that changing grating orientation had little effect on peak frequency despite robust changes in power. Our noise-masking manipulations are also novel and were important for showing that changes in gamma power and peak frequency need not involve changes in neuronal population response strength. Zhou et al. (2008) used a similar stimulus but with stationary rather than dynamic masking noise. Their data did not indicate a shift in peak frequency of gamma-modulated spiking activity. This could be due to a difference between the effects of static versus dynamic noise, or to gamma measurements of LFP power being more sensitive than those based on spiking activity (Zeitler et al., 2006).

Previous modeling work has provided a limited exploration of the relationship between these two properties of gamma. Whittington et al. (2011) summarized the relationship between gamma power and peak frequency suggested by several different models of gamma generation. In an interneuron network gamma model (ING), tonic excitation of an interneuron network generates gamma, with stronger drive producing greater power and higher peak frequency. In the PING model, perhaps the most relevant for cortical rhythms, gamma generation involves recurrent connections between interneurons and excitatory neurons. Gamma power and frequency increase with the strength of drive to either excitatory or inhibitory cells in the network (Traub et al., 1997). In the persistent gamma model, not typically invoked to explain cortical gamma (Bartos et al., 2007; Tiesinga and Sejnowski, 2009), an increase in gamma power does not require higher network firing rates nor does it lead to a change in peak frequency (Whittington et al., 2011). Thus, in their most straightforward instantiation, these models suggest peak frequency should increase with power (and network drive) or remain unchanged (Whittington et al., 2011), inconsistent with our findings.

We extended the basic PING architecture to include a feedback component and studied the activity level, gamma power, and gamma peak frequency it produced for a range of manipulations. The simulations were able to reproduce the physiological data, and suggested the importance of several distinct mechanisms. First, gamma peak frequency is modulated by a global input which could reflect long range horizontal interactions or feedback. This component is most prominent for large stimuli, and it enhances gamma power and lowers peak frequency. Similar to the effect of reducing stimulus size, Lima et al. (2010) reported that superimposing one grating on another reduces power but enhances peak frequency (see also Bartolo et al., 2011). Lima et al. (2010) suggest that this reflects competition between subsets of neurons activated by the two component gratings; in our formulation, such an effect could be similarly phrased as the withdrawal of a global, coordinating input when two gratings are presented.

We found that gamma peak frequency and power had different sensitivities to the strength of external input. Power decreased readily when input was weakened, but peak frequency was only altered when input was reduced substantially. Small changes in the time constant of the model had a nearly opposite influence: they strongly altered peak frequency, but had much weaker effect on gamma power. This is consistent with the behavior of ING models, in which longer inhibitory time constants result in lower gamma peak frequency (Traub et al., 1996a; Wang and Buzsáki, 1996), but can either increase or decrease network coordination (Wang and Buzsáki, 1996).

Finally, we show that a modulation of input strength and recurrent excitation can reduce both gamma power and peak frequency without altering response strength, as we observed in our noise masking experiments. We speculate that this may reflect the stronger synaptic depression of excitatory synapses to transient input. A recent study found that gamma peak frequency, measured with magnetoencephalography, is lower in individuals with larger cortical surface area (Schwarzkopf et al., 2012). These authors speculate that large cortical surface area may be associated with weaker coupling between columns, a mechanism similar to the weaker excitatory coupling we used to implement the effects of masking noise. We note that in the ING model, heterogeneous drive to inhibitory cells-as might occur with dynamic noise-reduces power (Wang and Buzsáki, 1996), but it is not clear whether this would also explain the drop in peak frequency. An alternative explanation for our noise masking data is that gamma is disrupted by transient input (Kruse and Eckhorn, 1996) and builds slowly after the abrupt onset of a stimulus (Ray and Maunsell, 2010; Jia et al., 2011). The weak gamma we observe with high levels of masking noise may thus be due to the disruption of the build-up of network gamma dynamics by noise that was renewed on every frame.

Previous modeling work has shown that gamma peak frequency is sensitive to the balance of excitation to inhibition (Brunel and Wang, 2003), with a higher ratio favoring a lower frequency. Further, recent measurements in human cortex suggest gamma frequency is lower when GABA concentration is depressed, although the relationship to inhibitory neuron activity is unclear (Edden et al., 2009). We did not explore the effect of altering the balance between excitation and inhibition in our model, beyond that caused by modulating recurrent excitation for our noise-masking simulations. It is certainly possible that our stimulus manipulations also altered the balance of excitation and inhibition in V1. However, whole-cell recordings in cat $\mathrm{V} 1$ show that the suppression of firing rate that occurs with larger grating stimuli involves a withdrawal of both excitatory and inhibitory input, relative to that seen in response to a small grating (Ozeki et al., 2009; see Haider et al., 2010 for contrary findings using transient presentations of natural scenes). Similarly, high contrast gratings of the preferred orientation cause an increase in both excitation and inhibition relative to low contrast or nonpreferred stimuli (Anderson et al., 2000; Priebe and Ferster, 2006). To our knowledge, similar measurements have not been made for masking noise. Thus, changes in stimulus size, contrast, and orientation appear to modulate excitation and inhibition similarly in local networks, although we cannot rule out some change in their balance.

Regardless of the network effects recruited by our stimulus manipulations, we show clearly that the mechanisms determining gamma power and peak frequency must be distinct, as these properties have no consistent relationship. Further, these properties should not be ascribed solely to network drive, the spatial extent of the activated ensemble, or the balance between excitation and inhibition, as suggested by previous studies. As a result of the dissociation of gamma power and peak frequency, only ensembles receiving visual input with matched contrast, noise perturbation, and size (or embedded in similar spatial contexts) would have a similar gamma peak frequency, and thus could maintain a consistent temporal relationship. Deviations in any of these properties would disrupt the ability of gamma to fulfill any of its proposed functions, even between ensembles with clearly elevated gamma power.

\section{References}

Albrecht DG, Hamilton DB (1982) Striate cortex of monkey and cat: contrast response function. J Neurophysiol 48:217-237. Medline

Anderson JS, Carandini M, Ferster D (2000) Orientation tuning of input conductance, excitation, and inhibition in cat primary visual cortex. J Neurophysiol 84:909-926. Medline

Angelucci A, Bressloff PC (2006) Contribution of feedforward, lateral and 
feedback connections to the classical receptive field center and extraclassical receptive field surround of primate V1 neurons. Prog Brain Res 154:93-120. CrossRef Medline

Atallah BV, Scanziani M (2009) Instantaneous modulation of gamma oscillation frequency by balancing excitation with inhibition. Neuron 62:566-577. CrossRef Medline

Bartolo MJ, Gieselmann MA, Vuksanovic V, Hunter D, Sun L, Chen X, Delicato LS, Thiele A (2011) Stimulus-induced dissociation of neuronal firing rates and local field potential gamma power and its relationship to the resonance blood oxygen level-dependent signal in macaque primary visual cortex. Eur J Neurosci 34:1857-1870. CrossRef Medline

Bartos M, Vida I, Jonas P (2007) Synaptic mechanisms of synchronized gamma oscillations in inhibitory interneuron networks. Nat Rev Neurosci 8:45-56. CrossRef Medline

Berens P, Keliris GA, Ecker AS, Logothetis NK, Tolias AS (2008) Feature selectivity of the gamma-band of the local field potential in primate primary visual cortex. Front Neurosci 2:199-207. CrossRef Medline

Börgers C, Kopell NJ (2008) Gamma oscillations and stimulus selection. Neural Comput 20:383-414. CrossRef Medline

Brunel N, Wang XJ (2003) What determines the frequency of fast network oscillations with irregular neural discharges? I. Synaptic dynamics and excitation-inhibition balance. J Neurophysiol 90:415-430. CrossRef Medline

Buia C, Tiesinga P (2006) Attentional modulation of firing rate and synchrony in a model cortical network. J Comput Neurosci 20:247-264. CrossRef Medline

Burns SP, Xing D, Shelley MJ, Shapley RM (2010a) Searching for autocoherence in the cortical network with a time-frequency analysis of the local field potential. J Neurosci 30:4033-4047. CrossRef Medline

Burns SP, Xing D, Shapley RM (2010b) Comparisons of the dynamics of local field potential and multiunit activity signals in macaque visual cortex. J Neurosci 30:13739-13749. CrossRef Medline

Burns SP, Xing D, Shapley RM (2011) Is gamma-band activity in the local field potential of V1 cortex a "clock" or filtered noise? J Neurosci 31: 9658-9664. CrossRef Medline

Buzsaki G (2006) Rhythms of the brain. New York: Oxford UP.

Carandini M, Heeger DJ, Movshon JA (1997) Linearity and normalization in simple cells of the macaque primary visual cortex. J Neurosci 17:8621-8644. Medline

Chalk M, Herrero JL, Gieselmann MA, Delicato LS, Gotthardt S, Thiele A (2010) Attention reduces stimulus-driven gamma frequency oscillations and spike field coherence in V1. Neuron 66:114-125. CrossRef Medline

Edden RA, Muthukumaraswamy SD, Freeman TC, Singh KD (2009) Orientation discrimination performance is predicted by GABA concentration and gamma oscillation frequency in human primary visual cortex. J Neurosci 29:15721-15726. CrossRef Medline

Engel AK, Fries P, Singer W (2001) Dynamic predictions: oscillations and synchrony in top-down processing. Nat Rev Neurosci 2:704-716. CrossRef Medline

Frien A, Eckhorn R, Bauer R, Woelbern T, Gabriel A (2000) Fast oscillations display sharper orientation tuning than slower components of the same recordings in striate cortex of the awake monkey. Eur J Neurosci 12:1453-1465. CrossRef Medline

Fries P (2009) Neuronal gamma-band synchronization as a fundamental process in cortical computation. Annu Rev Neurosci 32:209-224. CrossRef Medline

Fries P, Reynolds JH, Rorie AE, Desimone R (2001) Modulation of oscillatory neuronal synchronization by selective visual attention. Science 291: 1560-1563. CrossRef Medline

Gawne TJ, Kjaer TW, Richmond BJ (1996) Latency: another potential code for feature binding in striate cortex. J Neurophysiol 76:1356-1360. Medline

Gieselmann MA, Thiele A (2008) Comparison of spatial integration and surround suppression characteristics in spiking activity and the local field potential in macaque V1. Eur J Neurosci 28:447-459. CrossRef Medline

Gray CM, König P, Engel AK, Singer W (1989) Oscillatory responses in cat visual cortex exhibit inter-columnar synchronization which reflects global stimulus properties. Nature 338:334-337. CrossRef Medline

Gupta A, Wang Y, Markram H (2000) Organizing principles for a diversity of GABAergic interneurons and synapses in the neocortex. Science 287: 273-278. CrossRef Medline

Haider B, Krause MR, Duque A, Yu Y, Touryan J, Mazer JA, McCormick DA
(2010) Synaptic and network mechanisms of sparse and reliable visual cortical activity during nonclassical receptive field stimulation. Neuron 65:107-121. CrossRef Medline

Henrie JA, Shapley R (2005) LFP power spectra in V1 cortex: the graded effect of stimulus contrast. J Neurophysiol 94:479-490. CrossRef Medline

Jia X, Kohn A (2011a) Gamma rhythms in the brain. PLoS Biol 9:e1001045. CrossRef Medline

Jia X, Smith MA, Kohn A (2011b) Stimulus selectivity and spatial coherence of gamma components of the local field potential. J Neurosci 31:9390-9403. CrossRef Medline

Kang K, Shelley M, Henrie JA, Shapley R (2010) LFP spectral peaks in V1 cortex: network resonance and cortico-cortical feedback. J Comput Neurosci 29:495-507. CrossRef Medline

Kruse W, Eckhorn R (1996) Inhibition of sustained gamma oscillations $(35-80 \mathrm{~Hz})$ by fast transient responses in cat visual cortex. Proc Natl Acad Sci U S A 93:6112-6117. CrossRef Medline

Lima B, Singer W, Chen NH, Neuenschwander S (2010) Synchronization dynamics in response to plaid stimuli in monkey V1. Cereb Cortex 20: 1556-1573. CrossRef Medline

Mazzoni A, Panzeri S, Logothetis NK, Brunel N (2008) Encoding of naturalistic stimuli by local field potential spectra in networks of excitatory and inhibitory neurons. PLoS Comput Biol 4:e1000239. CrossRef Medline

Ozeki H, Finn IM, Schaffer ES, Miller KD, Ferster D (2009) Inhibitory stabilization of the cortical network underlies visual surround suppression. Neuron 62:578-592. CrossRef Medline

Pesaran B, Pezaris JS, Sahani M, Mitra PP, Andersen RA (2002) Temporal structure in neuronal activity during working memory in macaque parietal cortex. Nat Neurosci 5:805-811. CrossRef Medline

Priebe NJ, Ferster D (2006) Mechanisms underlying cross-orientation suppression in cat visual cortex. Nat Neurosci 9:552-561. CrossRef Medline

Ray S, Maunsell JH (2010) Differences in gamma frequencies across visual cortex restrict their possible use in computation. Neuron 67:885-896. CrossRef Medline

Ray S, Maunsell JH (2011) Different origins of gamma rhythm and highgamma activity in macaque visual cortex. PLoS Biol 9:e1000610. CrossRef Medline

Roelfsema PR, Lamme VA, Spekreijse H (2004) Synchrony and covariation of firing rates in the primary visual cortex during contour grouping. Nat Neurosci 7:982-991. CrossRef Medline

Schwarzkopf DS, Robertson DJ, Song C, Barnes GR, Rees G (2012) The frequency of visually induced gamma-band oscillations depends on the size of early human visual cortex. J Neurosci 32:1507-1512. CrossRef Medline

Singer W (1999) Neuronal synchrony: a versatile code for the definition of relations? Neuron 24: 49-65:111-125.

Thomson AM, Deuchars J (1997) Synaptic interactions in neocortical local circuits: dual intracellular recordings in vitro. Cereb Cortex 7:510-522. CrossRef Medline

Tiesinga P, Sejnowski TJ (2009) Cortical enlightenment: are attentional gamma oscillations driven by ING or PING? Neuron 63:727-732. CrossRef Medline

Traub RD, Whittington MA, Colling SB, Buzsáki G, Jefferys JG (1996a) Analysis of gamma rhythms in the rat hippocampus in vitro and in vivo. J Physiol 493:471-484. Medline

Traub RD, Whittington MA, Stanford IM, Jefferys JG (1996b) A mechanism for generation of long-range synchronous fast oscillations in the cortex. Nature 383:621-624. CrossRef Medline

Traub RD, Jefferys JG, Whittington MA (1997) Simulation of gamma rhythms in networks of interneurons and pyramidal cells. J Comput Neurosci 4:141-150. CrossRef Medline

Wang XJ, Buzsáki G (1996) Gamma oscillation by synaptic inhibition in a hippocampal interneuronal network model. J Neurosci 16:6402-6413. Medline

Whittington MA, Traub RD, Jefferys JG (1995) Synchronized oscillations in interneuron networks driven by metabotropic glutamate receptor activation. Nature 373:612-615. CrossRef Medline

Whittington MA, Cunningham MO, LeBeau FE, Racca C, Traub RD (2011) Multiple origins of the cortical gamma rhythm. Dev Neurobiol 71:92-106. CrossRef Medline

Wilson HR, Cowan JD (1972) Excitatory and inhibitory interactions in lo- 
calized populations of model neurons. Biophys J 12:1-24. CrossRef Medline

Womelsdorf T, Fries P, Mitra PP, Desimone R (2006) Gamma-band synchronization in visual cortex predicts speed of change detection. Nature 439:733-736. CrossRef Medline

Xing D, Yeh CI, Burns S, Shapley RM (2012) Laminar analysis of visually evoked activity in the primary visual cortex. Proc Natl Acad Sci U S A 109:13871-13876. CrossRef Medline

Zanos TP, Mineault PJ, Pack CC (2011) Removal of spurious correlations between spikes and local field potentials. J Neurophysiol 105:474-486. CrossRef Medline

Zeitler M, Fries P, Gielen S (2006) Assessing neuronal coherence with single-unit, multi-unit, and local field potentials. Neural Comput 18: 2256-2281. CrossRef Medline

Zhou Z, Bernard MR, Bonds AB (2008) Deconstruction of spatial integrity in visual stimulus detected by modulation of synchronized activity in cat visual cortex. J Neurosci 28:3759-3768. CrossRef Medline 